J. Clin. Chem. Clin. Biochem.

Vol. 14, 1976, pp. 9-13

\title{
Stabilisierung des reduzierten $\beta$-Nicotinamid-Adenin-Dinucleotid in einem organischen Lösungsmittel
}

\author{
Von H. Gallati
}

Diagnostische Forschungsabteilung der F. Hoffmann-La Roche \& Co. AG, Basel

(Eingegangen am 21. August/23.. Oktober 1975)

Zusammenfassung: Das NADH zeigt eine erstaunlich gute Lagerstabilität, sofern dieses Coenzym nicht in einem wäßrigen - wie bisher -, sondern in einem wasserfreien, organischen Lösungsmittel gelöst wird.

In der vorliegenden Arbeit werden die Resultate über die Stabilität des NADH mitgeteilt, das in Ethylenglykol-Tris gelöst und während 136 Tagen bei den Temperaturen $2-8^{\circ} \mathrm{C}, 19-22^{\circ} \mathrm{C}, 35^{\circ} \mathrm{C}, 45^{\circ} \mathrm{C}$ und $60^{\circ} \mathrm{C}$ inkubiert wurde. Zum Vergleich wurde parallel dazu NADH in dest. Wasser gelöst und in gleicher Weise die Stabilität geprüft.

\section{Stabilization of reduced $\beta$-nicotinamide-adenine-dinucleotide in an organic solvent}

Summary: NADH is surprisingly stable, providing it is stored in anhydrous organic solvents, and not, as hitherto, in aqueous solvents. The stability of NADH was investigated in solution in ethylene glycol-Tris over a period of 136 days at temperatures of $2-8^{\circ} \mathrm{C}, 19-22^{\circ} \mathrm{C}, 35^{\circ} \mathrm{C}, 45^{\circ} \mathrm{C}$ and $60^{\circ} \mathrm{C}$. For comparison, the stability of NADH in solution in distilled water was studied under similar conditions.

\section{Einleitung}

Das Problem der Stabilisierung des NADH wurde in zahlreichen Arbeiten untersucht. Dabei ist zu beachten, daß die Instabilität des NADH sich nicht nur auf die reduzierte Form, sondern auch auf die Bildung eines Inhibitors bezieht, der schon in kleinsten Mengen die Aktivitätsbestimmung der Oxidoreductasen wesentlich zu stören vermag.

NADH zeigt eine gute Lagerstabilität, sofern das Salz unter Ausschluß von Feuchtigkeit und oxydierenden Substanzen aufbewahrt wird. Daher sollte es eher in kleinen Portionen (z. B. , ,Tages-Portionen ") konfektioniert werden $(1-2)$.

NADH in Lösung gilt allgemein als instabil. Zur Verbesserung der Stabilitä̆t wurde der Einfluß verschiedener Puffersysteme, des pH-Wertes der NADH- Lösung, von Anionen und Kationen sowie der NADH-Konzentration bei einzelnen Lagertemperaturen untersucht $(1-8)$. $\mathrm{Da}$ bei haben sich folgende Bedingungen als vorteilhaft erwiesen: $10 \mathrm{mmol} / \mathrm{l} \mathrm{NADH}$ in $50 \mathrm{mmol} / 1$ Tris-EDTA-HCl, $\mathrm{pH} 7,7$. Unter diesen Bedingungen ist das NADH bei Raumtemperatur $1 \mathrm{Tag}$, und bei $2-8^{\circ} \mathrm{C} 1$ Woche stabil (1).

Eine entscheidende Stabilitätsverbesserung des NADH, das unseres Wissens bisher stets in wạßßrigem Milieu ge- löst wurde, konnte trotz vieler Versuche bisher nicht erreicht werden.

Wenn man aber bedenkt, daß das NADH-Salz eine gute Stabilität aufweist, sofern durch Ausschluß von Wasser und Sauerstoff die Autoxydation des NADH wie auch die Bildung des Oxidoreductase-Inhibitors unterbunden wird, so drängt sich der Versuch auf, das NADH in einem wasserfreien, organischen Lösungsmittel zu lösen und unter diesen neuen Bedingungen die Stabilität des gelösten NADH zu prüfen.

\section{Material und Methoden}

\section{Material}

Alle organischen Lösungsmittel waren von analytischer Reinheit. Ethylenglykol (1,2-Ethandiol, Glycol puriss. p.a. 99,5\%, MG: $\left.62,07, \mathrm{~F}:-11--9^{\circ} \mathrm{C}, \mathrm{Kp}: 92^{\circ} \mathrm{C}, \mathrm{d}_{4}^{20}: 1.113, \mathrm{n}_{\mathrm{D}}^{20}: 1.431\right)$ wurde von Fluka bezogen, Methanol, 1-Propanol und Aceton waren von Merck.

Als Puffersubstanz wurde Tris-(hydroxymethyl)-aminomethan (Tris, Fluka, puriss. p.a. MG: 121,14) eingesetzt.

NAD und NADH wurde von Boehringer-Mannheim geliefert. Beide Coenzyme waren vom Reinheitsgrad II.

Als Enzymkontrollserum wurde ein eigenes, Albumin-haltiges, lyophilisiertes Enzympräparat eingesetzt, das unmittelbar vor Gebrauch durch Zugabe von dest. Wasser rekonstituiert wurde. Die Werte für die einzelnen Enzyme lagen im leicht pathologischen Bereich. 


\section{Methoden}

Die Zusammensetzung der Testlösung wie auch die Testbedingungen zur Aktivitätsbestimmung der einzelnen Enzyme entsprach mit Ausnahme der speziell erwähnten Zusätze - den Empfehlungen der Deutschen Enzymkommission (9).

Zur Bestimmung der gebildeten Menge Oxidoreductase-Inhibitor wurden zur Lactat-Dehydrogenase-Testlösung eine bestimmte Konzentration des inkubierten Coenzyms zugemischt und unter diesen Bedingungen die Lactat-Dehydrogenase-Aktivität gemessen.

Für die Stabilitätsprüfung der NADH-Lösung mußten zwei Gesichtspunkte berücksichtigt werden:

1. Die Stabilität der reduzierten Form des NADH. Dazu wurden zu 2,5 ml Lactat-Dehydrogenase-Testlösung $0,1 \mathrm{ml}$ der zu prüfenden NADH-Lösung, die während einer gewissen Zeitdauer bei einer bestimmten Temperatur inkubiert wurde, zugemischt und die Extinktion dieser Lösung bei der Wellenlänge $366 \mathrm{~nm}$ gemessen. Die jeweils gemessenen Werte werden in Prozent des anfänglichen NADH-Gehaltes ausgedrückt.

2. Die Bildung des Oxidoreductase-Inhibitors: Dazu wurden zur obigen Lactat-Dehydrogenase-Testlösung $0,1 \mathrm{ml}$ des frisch rekonstituierten Enzymkontrollserum zupipettiert und die Extinktionsdifferenz $(\Delta \mathrm{E} / \mathrm{min}$.) bei der Wellenlänge $366 \mathrm{~nm}$ und der Inkubationstemperatur von $25^{\circ} \mathrm{C}$ bestimmt. Da aber die Lactat-Dehydrogenase-Aktivität nicht nur vom Vorhandensein des Oxidoreductase-Inhibitors, sondern.auch von der NADHKonzentration abhängt, wurde nach 136 Fagen Inkubation bei jenen Proben, die nur noch wenig NADH enthielten, frisches NADH zugefügt und mit diesen Lösungen die LactatDehydrogenase-Aktivität gemessen. Die jeweils erhaltenen Werte der Extinktionsdifferenz ( $\Delta \mathrm{E} / \mathrm{min}$ ) werden in Prozent der anfänglichen Lactat-Dehydrogenase-Aktivität ausgedrückt.

\section{Resultate}

NADH in wäßriger Lösung muß auch deswegen als instabil angesehen werden, weil sich ein OxidoreductaseInhibitor bildet, der schon in kleinsten Konzentrationen die Aktivitätsbestimmung der Oxidoreductasen zu stören vermag.

Dieser Inhibitor bildet sich nicht aus dem NADH, sondern nur aus dem NAD, das im NADH-Präparat schon enthalten ist oder durch Autoxydation des NADH entsteht (2).

Daher wurden in einem Vorversuch je $10 \mathrm{mg}$ NAD zu $1 \mathrm{ml}$ der folgenden Lösungsmittel zugegeben: Methanol$50 \mathrm{mmol} / 1$ Tris, 1 -Propanol-50 mmol/l Tris, Aceton$50 \mathrm{mmol} / \mathrm{l}$ Tris, Ethylenglykol-50 mmol/1 Tris und zum Vergleich 0,1 mol/1 Phosphatpuffer, pH 10,5. Die Substanzmischungen wurden in verschlossenen Glasampullen 1 Tag lang bei $45^{\circ} \mathrm{C}$ inkubiert. Anschließend wurde die Aktivität der Lactat-Dehydrogenase bestimmt, indem zur Testlösung $100 \mu \mathrm{mol} / 1$ des inkubierten NAD zugefügt wurde. Während in den organischen NAD-Lösungen keine Inhibitorbildung festgestellt werden konnte, ergab das NAD, das in Phosphatpuffer inkubiert wurde, eine Aktivitặtshemmung der Lactat-Dehydrogenase von $85 \%$.

Nachdem weitere Vorversuche zeigten, daß die Aktivitätsbestimmung diagnostisch wichtiger Enzyme (Lactat-Dehydrogenase-1-Isoenzym, Glutamat-Dehydrogenase, Glutamat-Pyruvat- und Glutamat-Oxalacetat-Transaminase und Creatin-Kinase) durch das Lösungsmittel Ethylenglykol. $50 \mathrm{mmol} / \mathrm{l}$ Tris in der fraglichen Konzentration (bis $0,1 \mathrm{ml} / 2,7 \mathrm{ml}$ Testlösung) nicht beeinflußt wird, wurde für die weiteren Versuche dieses Lösungsmittel eingesetzt.

In $25 \mathrm{ml}$ Ethylenglykol wurden bei Raumtemperatur und unter leichtem Rühren $150 \mathrm{mg}$ Tris und anschließend $4,5 \mathrm{mg} / \mathrm{ml}$ NADH-Dinatriumsalz (NADH-Gehalt $=80 \%$, NADH-Konzentration $=5,4 \mathrm{mmol} / \mathrm{l})$ gelöst.

Für einen Stabilitätsvergleich wurde parallel dazu die gleiche Menge NADH-Dinatriumsalz von der selben Charge in dest. Wasser gelöst. Nach Durchführung der Anfangsanalysen (Bestimmung der Extinktion bei $366 \mathrm{~nm}$ und der Aktivität der Lactat-Dehydrogenase, vgl. Methoden) wurden die beiden NADH-Lösungen in je 5 Glasampullen mit Schraubverschluß aufgeteilt und bei den Temperaturen $2-8^{\circ} \mathrm{C}, 19-22^{\circ} \mathrm{C}, 35^{\circ} \mathrm{C}, 45^{\circ} \mathrm{C}$ und $60^{\circ} \mathrm{C}$ inkubiert. Nach bestimmten Zeitintervallen wurden mit den einżelnen NADH-Lösungen von neuem die Extinktion
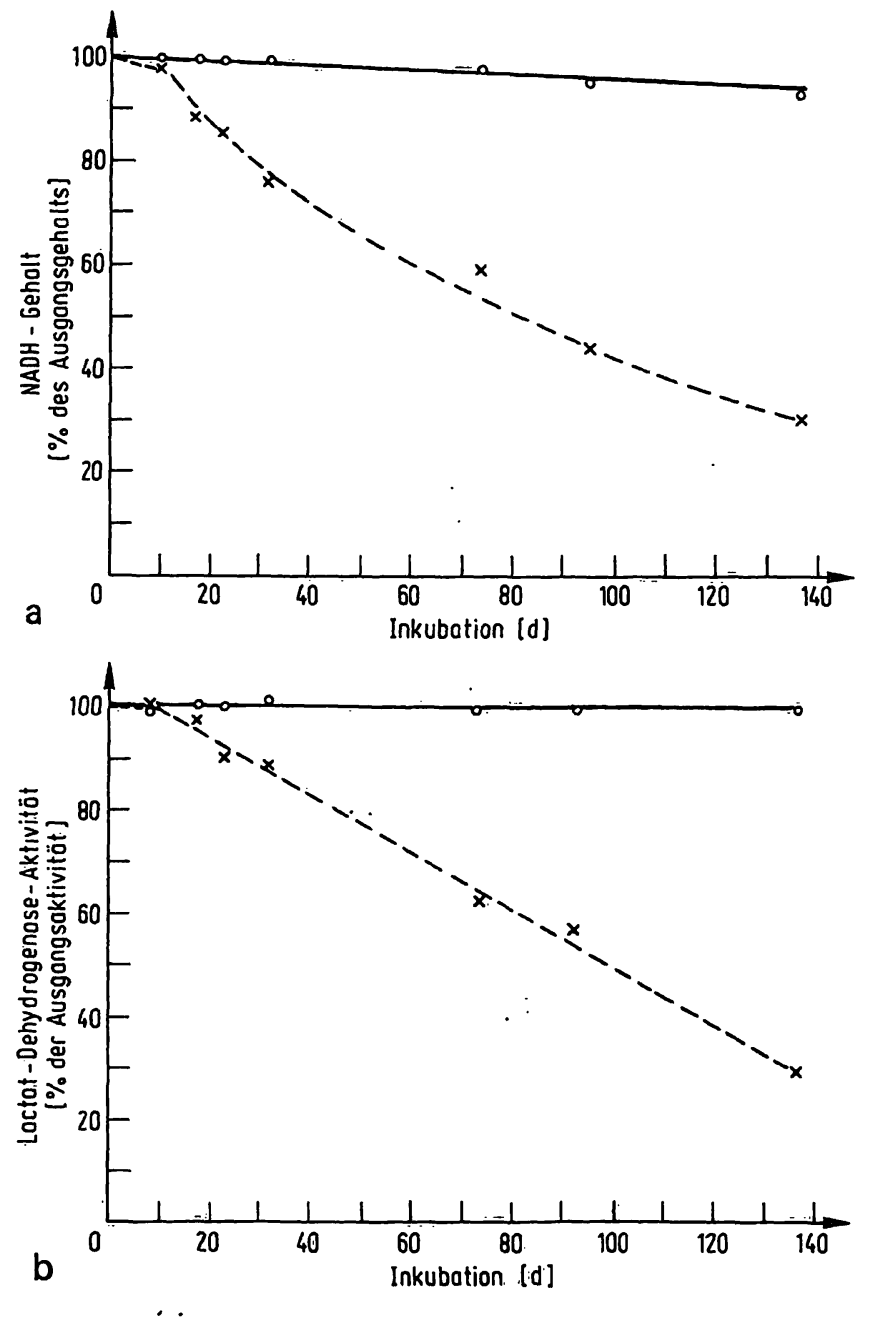

Abb. 1. Stabilität der NADH-Lösung bei $2-8^{\circ} \mathrm{C}$

Nach 136 Tagen beträgt der NADH-Gehalt (Abb. 1) in Ethylenglykol-50 mmol/1 Tris (o- 0 ) noch immer $92 \%$, während in dest. Wasser $(x---x)$ nur noch $29 \%$ des eingesetzten NADH vorhanden sind.

Wird mit diesen beiden NADH-Lösungen die Aktivität der Lactat-Dehydrogenase (Abb. 1 b) im Enzymkontrollserum gemessen, so erhält man $100 \%$ Aktivität, wenn das NADH in Ethylenglykol-50 mmol/1 Tris gelöst wurde, in dest. Wasser aber beträgt die Aktivität noch $\mathbf{3 0 \%}$. 
bei 366 nm sowie die Aktivität der Lactat-Dehydrogenase gemessen.

Die Resultate der Stabilitätsprüfung sind in der Weise dargestellt, daß die jeweils gemessene Extinktion und die Aktivität der Lactat-Dehydrogenase in Prozent der Anfangswerte ausgedrückt und in den Abbildungen 1-4 graphisch aufgezeichnet sind.

Diese Versuche zeigen, daß NADH, das in Ethylenglykol$50 \mathrm{mmol} / 1$ Tris gelöst wird, einige Monate bei $2-8^{\circ} \mathrm{C}$ und auch bei Raumtemperatur aufbewahrt werden kann, ohne daß die Aktivitätsbestimmung der Lactat-Dehydrogenase gestört wird.
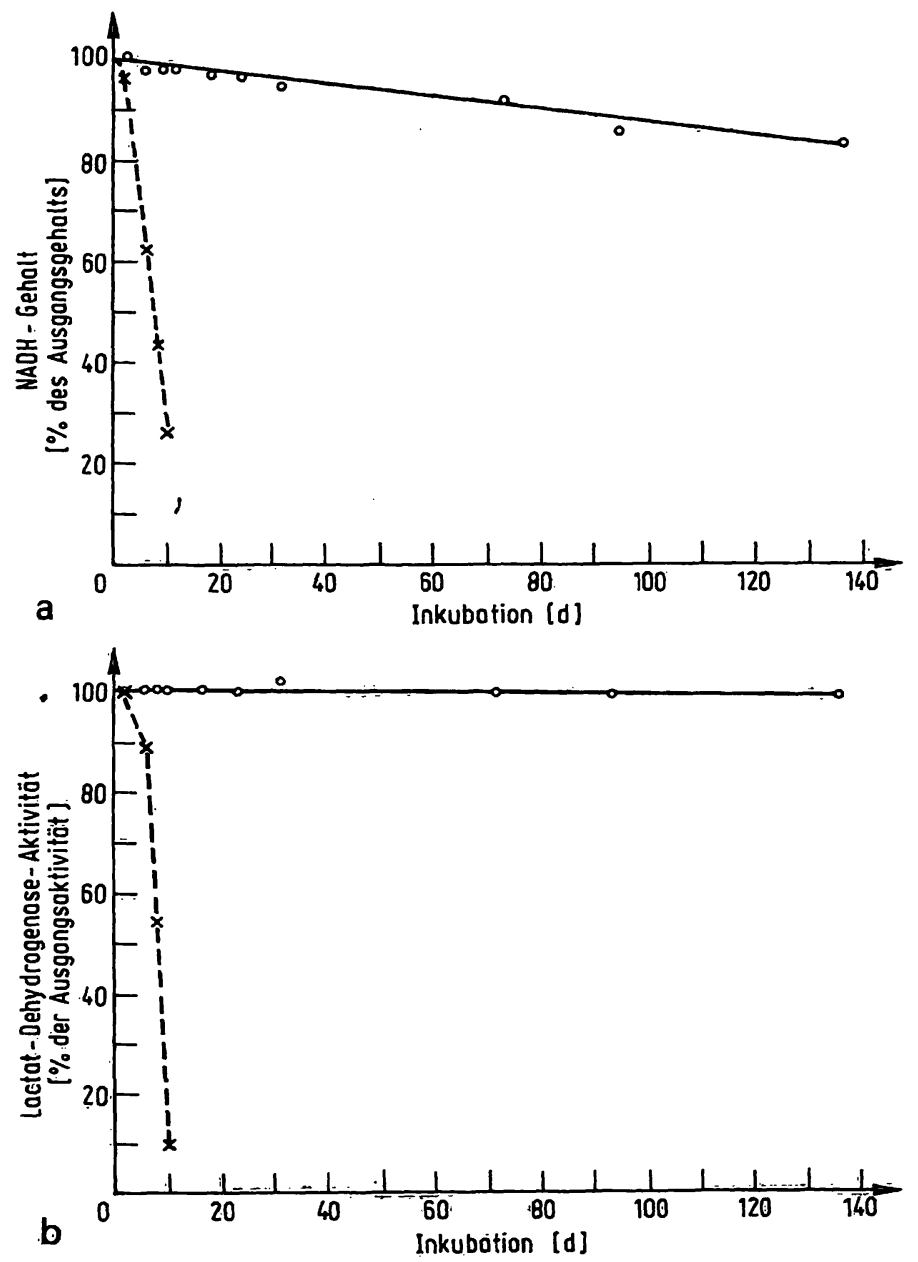

Abb. 2. Stabilität der NADH-Lösung bei $19-22^{\circ} \mathrm{C}$ Schon innèrhalb von 10 Tagen sinkt der NADH-Gehalt (Ảbb. 2a) in der wäßrigen Lösung $(x-=-x)$ auf $26 \%$, während in der Ethylenglykol-50 mmol/1 Tris-Lösung $(\circ-0)$ nach 136 Tagen noch immer $83 \%$ des ursprüng lich vorhandenen NADH enthalten sind.

Entsprechend sinkt auch die Aktivität der Lactat-Dehydrogenase (Abb. 1b) mit der wäßrigen NADH-Lösung nach 10 Tagen Inkubation auf $9 \%$. Die Aktivität mit dem NADH, das in Ethylenglykol-50 mmol/1 Tris gelöst und 136 Tage bei $19-22^{\circ} \mathrm{C}$ inkubiert wurde, beträgt noch 98\%.
Anschließend wurden die während 136 Tagen inkubierten NADH-Lösungen auf einen möglichen Gehalt an Oxidoreductase-Inhibitor untersucht. Dazu wurde die Aktivität der Lactat-Dehydrogenase bestimmt, nachdem der Testlösung $200 \mu \mathrm{mol} / 1$ des während 136 Tagen inkubierten NADH zugefügt wurden. Während im NADH, das in Ethylenglykol-50 mmol/1 Tris gelöst und bei den Temperaturen von $2-8^{\circ} \mathrm{C}, 19-22^{\circ} \mathrm{C}, 35^{\circ} \mathrm{C}$ und $45^{\circ} \mathrm{C}$ inkubiert wurde, keine Inhibitorbildung festgestellt werden konnte, wurde mit der wäßrigen NADH-Lösung, die bei $2-8^{\circ} \mathrm{C}$ aufbewahrt wurde, die Aktivität der Lactat-Dehydrogenase zu $21 \%$ gehemmt. (Die NADH-Lösun-
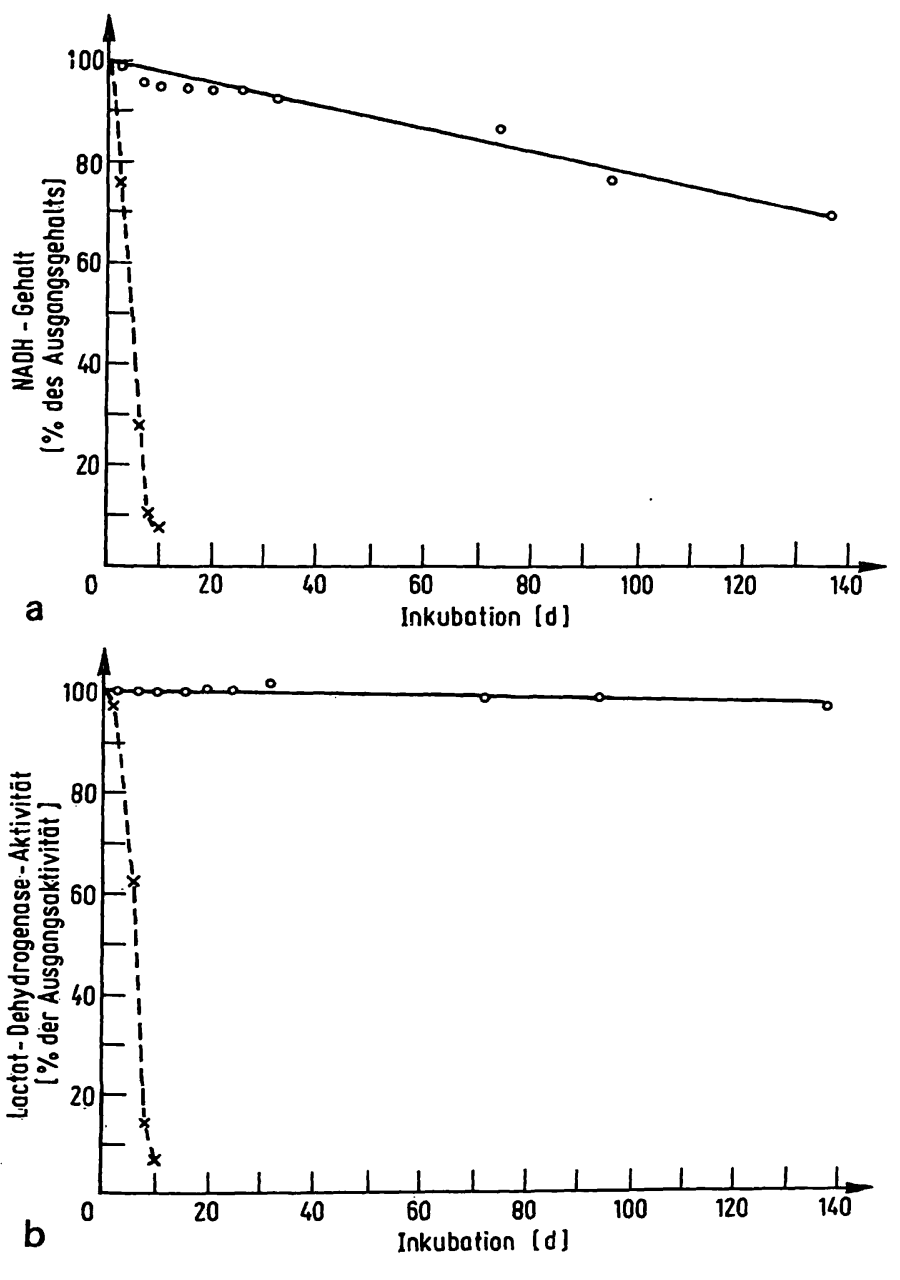

Abb. 3. Stabilität der NADH-Lösung bei $35^{\circ} \mathrm{C}$

Die Abnahme des NADH-Gehaltes (Abb. 3a) in der wäßrigen Lösung $(\mathbf{x}---\mathrm{x})$ geht bei der Inkubationstemperatur von $35^{\circ} \mathrm{C}$ noch schneller vor sich (nach 8 Tagen sind noch $11 \%$ NADH vorhanden), während das NADH in Ethylenglykol-50 mmol/1 Tris (o- -0$)$ nach 136 Tagen noch immer zu $69 \%$ vorhanden ist.

Die Aktivitätsbestimmung der Lactat-Dehydrogenase (Abb. 3b) mit der wäßrigen NADH-Lösung ergibt nach 10 Tagen Inkubation 7\% des anfänglichen Wertes. Mit der Ethylenglykol-50 mmol/1 Tris-NADH-Lösung wird nach einer 136-tägigen Inkubation noch $97 \%$ der ursprụ̈nglichen Lactat-Dehydrogenase-Aktivität gemessen. 
gen der übrigen Inkubationstemperaturen wurden zu einem.früheren Zeitpunkt verworfen und konnten daher nicht in diese Versuche mit einbezogen werden.)
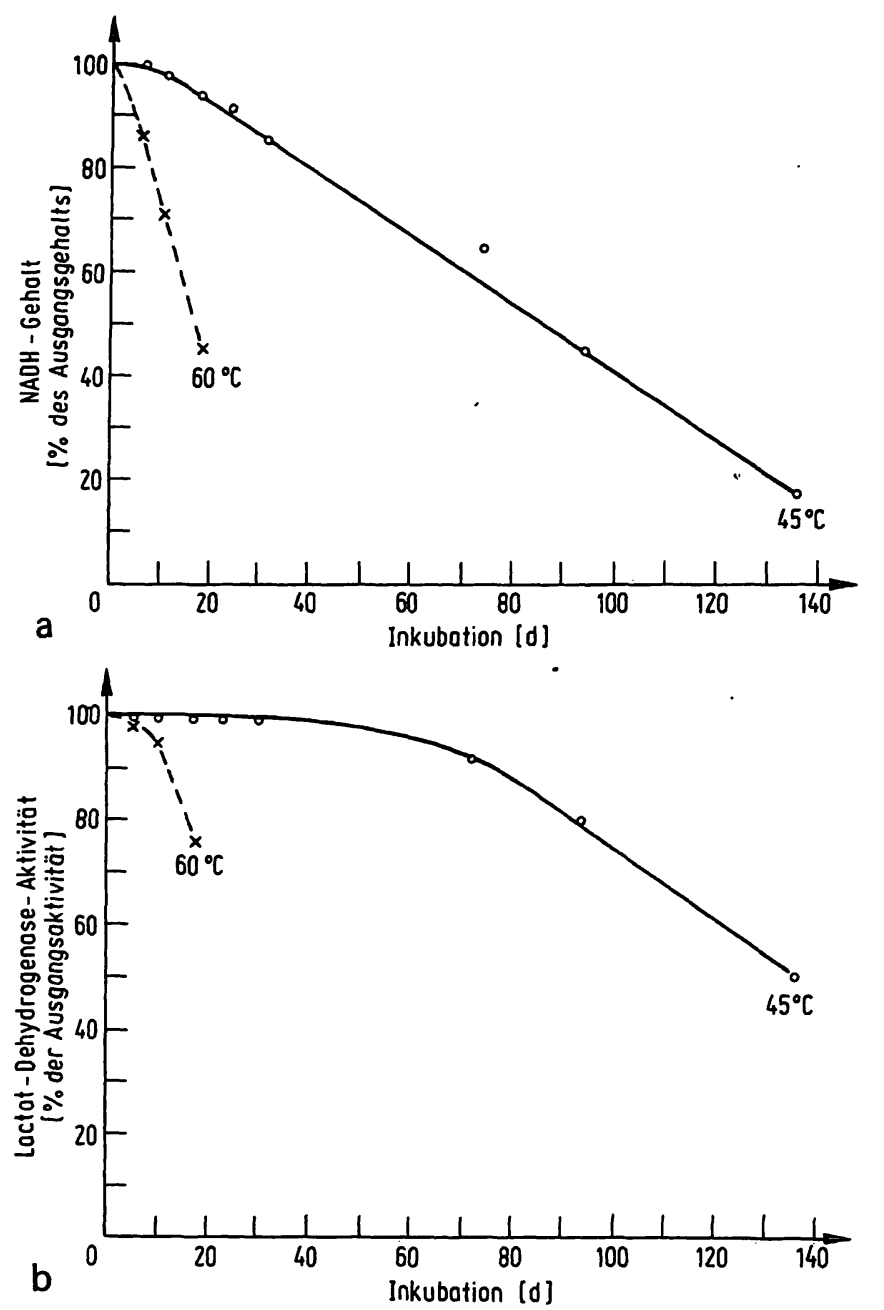

Abb. 4. Stabilität der NADH-Lösung bei $45^{\circ} \mathrm{C}$ und $60^{\circ} \mathrm{C}$ $\mathrm{Da}$ bei diesen Inkubationstemperaturen das NADH in dest. Wasser schon nach wenigen Stunden vollständig zerstört war und damit keine Lactat-Dehydrogenase-Aktivivität mehr gemessen werden konnte, wurde bei der Darstellung dieser Abbildung nur die Stabilität des NADH, das in Ethylenglykol-50 mmol/1 Tris gelöst wurde, berücksichtigt.

Bei der Inkubationstemperatur von $45^{\circ} \mathrm{C}(\mathrm{O}-\mathrm{O})$ beträgt der NADH-Gehalt (Abb. $4 \mathrm{a}$ ) nach 136 Tagen noch $17 \%$, während bei $60^{\circ} \mathrm{C}(\mathrm{x}-\mathrm{x})$ nach 17 Tagen noch $46 \%$ des ursprünglichen NADH vorhanden sind.

Die Bestimmung der Lactat-Dehydrogenase-Aktivität (Abb. 4b) mit der entsprechenden NADH-Lösung ergibt folgende Werte: Bei $45^{\circ} \mathrm{C}$ inkubiert nach 136 Tagen noch $49 \%$, und bei $60^{\circ} \mathrm{C}$ inkubiert nach 17 Tagen $76 \%$ der anfänglichen Aktivität

\section{Diskussion}

Auf Grund der in dieser Arbeit mitgeteilten Resultate kann gesagt werden, daß NADH, das in einem wasserfreien, organischen Lösungsmittel aufgenommen wird, eine gute Lagerstabilität zeigt.

Bei der Wahl eines geeigneten organischen Lösungsmittels müssen die folgenden Punkte berücksichtigt werden:

1. das NADH muß löslich sein

2. das NADH darf durch das Lösungsmittel oder irgendwelche Zusätze nicht oxydiert werden

3. das Lösungsmittel dạrf die entsprechenden enzymatischen Tests nicht beeinflussen

4. das Lösungsmittel muß bezüglich Testdurchführung gute Praktikabilität aufweisen (Viskosität, Dampfdruck, hygroskopische Eigenschaft, Mischbarkeit mit der entsprechenden Testlösung).

Ohne die Einsatzmöglichkeit anderer organischer Lösungsmittel auszuschließen, wurde für die eingehende Stabilitätsprüfung des gelösten NADH dạs Lösungsmittel Ethylenglykōl-50 mmol/1 Tris gewählit, da dieses die oben angefuhrrten Voraussetzungen erfüllt.

Die Stabilität der wäßrigen NADH-Lösung stimmt in etwa mit den Stabilitätsresultaten überein, wie sie kürzlich von Gerhardt (1) veröffentlicht wurden. Auf Grund ausgedehnter Versuche hat Gerhardt vorgeschlagen, das NADH in $50 \mathrm{mmol} / 1$ Tris-EDTA-HCl-Puffer, $\mathrm{pH} 7,7$ $\left(25^{\circ} \mathrm{C}\right)$ zu lösen. Für die Lagerstabilität dieser gepufferten NADH-Lösung erhielt er folgende Resultate: Bei Raumtemperatur aufbewahrt, waren nach 4 Tagen noch $50 \%$ des anfänglichen NADH-Gehaltes vorhanden, und die Aktivitätsbestimmung der Lactat-Dehydrogenase mit dieser Coenzymlösung ergab noch $70 \%$ des ursprünglichen Wertes. Wurde die NADH-Lösung bei $4^{\circ} \mathrm{C}$ gelagert, waren nach 14 Tagen noch $65 \%$ NADH vorhanden und ergaben $85 \%$ der anfänglichen Lactat-Dehydrogenase-Aktivität. Auf Grund der bisher veröffentlichten Arbeiten (1-8) ist anzunehmen, daß auch unter optimalen Bedingungen die Stabilität einer wäßrigen NADH-Lösung nicht wesentlich verbessert werden kann. Dies ist aber möglich, sofern das NADH in einem wasserfreien, organischen Lösungsmittel aufgenommen wird.

Für die manuelle Testausführung von NADH-abhängigen Analysen hat sich eine Tropfflasche bewährt, in der das $\mathrm{NADH}$ - in Ethylenglykol-50 mmol/1 Tris gelöst - enthalten ist. Zum Starten der Reaktion werden zwei Tropfen davon 'der Testlösung zugemischt. 


\section{Literatur}

1. Gerhardt, W., Kofoed, B., Westlund, L. \& Pavlu, B. (1974), Scand. .J. Clin. Lab. Invest. 33, 3-51.

2. Gallati, H. (1976), diese Z. 14, 3-8.

3. Winer, A. \& Schwert, G. (1958), J. Biol. Chem. 231, 10651083.

4. Stock, A., Sann, E. \& Pfleiderer, G. (1961), Justus Liebigs Ann. Chem. 647, 188-219.

5. Lowry, O., Passonneau, J. \& Rock, M. (1961), J. Biol. Chem. 236, 2756-2759.
6. Alivisatos, S., Ungar, F. \& Abraham, G. (1964), Nature (London) 203, 973-975.

7. Strandjord, P. \& Clayson, K. (1966), J. Lab. Clin. Med. 67, $144-153$.

8. James, J., Johnson, B. \& Schaffer, R. (1972), Proc. Int. Seminar and Workshop on Enzymology, Chicago.

9. Deutsche Gesellschaft für Klinische Chemie (1972), diese Z. 10, 182-192.
Dr. H. Gallati,

Diagnostische Forschungsabteilung,

F. Hoffmann-La Roche \& Co. AG,

Grenzacherstr. 124,

CH-4002 Basel, Schweiz 
